\title{
Adsorption of Methanol on the MgO(100) Surface: An Infrared Study at Room Temperature
}

\author{
Jennifer Rudberg and Michelle Foster* \\ Department of Chemistry, University of Massachusetts-Boston, 100 Morrissey Boulevard, \\ Boston, Massachusetts 02125
}

Received: July 14, 2004; In Final Form: September 15, 2004

\begin{abstract}
Methanol adsorbed on the (100) face of $\mathrm{MgO}$ has been studied at room temperature and elevated methanol pressures using transmission Fourier transform infrared spectroscopy. From these investigations, we find evidence of two structures for the adsorbed methanol. At low coverages, the methanol molecules congregate into two-dimensional islands that are strongly associated with the substrate. The properties of this submonolayerto-monolayer thin film are similar to those of solid methanol. Once the available surface sites are occupied and a complete monolayer has formed, the methanol begins to condense in multilayers, creating a thin film on the surface with properties similar to liquid methanol.
\end{abstract}

\section{Introduction}

Magnesium oxide is often described as a model basic oxide, having a simple rock salt structure, a single valence state, and only one stable low-index surface orientation: the (100) face. $\mathrm{MgO}$ has been shown to catalyze the hydrogenation of 1,3butadiene, the $\mathrm{H}_{2}$ and $\mathrm{D}_{2}$ exchange reaction, and the dehydrogenation of methanol and formic acid. ${ }^{1}$ As such, it is well suited to fundamental studies of adsorption and catalysis at oxide surfaces. The surface chemistry and physics of metal oxides, and the characterization of adsorbates on these materials, are important to many natural and industrial processes. ${ }^{2,3}$ Despite the evident need, few surface spectroscopic studies have been reported on well-characterized mineral surfaces. Such studies have been hampered by the inherent complexity of oxide surfaces and the experimental difficulty of using electron and ion spectroscopies for the analysis of insulating materials. As a result, although they are essential for a more complete understanding of reactions on oxide surfaces at the molecular level, studies using a surface science approach are limited, and the investigation of magnesium oxide adsorption systems has been confined, in general, to the less well-defined surfaces of powders. ${ }^{4-6}$ To avoid the problems associated with analysis of single-crystal samples of an insulating material, a number of studies have made use of thin films of well-characterized $\mathrm{MgO}$ (100) grown on a conducting substrate, typically either $\operatorname{Mo}(100)^{7-10}$ or $\operatorname{Mg}(0001) .{ }^{11,12}$ However, the use of optically based spectroscopy such as transmission infrared (IR) spectroscopy avoids the use of charged particles altogether. ${ }^{13,14}$

The adsorption of methanol on magnesium oxide has been investigated on powder samples, well-characterized thin films, and single-crystal substrates. ${ }^{4-6,15}$ The interaction of methanol with magnesium oxide powders has been studied with IR spectroscopy, ${ }^{4,6}$ temperature-programmed desorption (TPD) mass spectrometry, ${ }^{5}$ and nuclear magnetic resonance (NMR) spectroscopy ${ }^{15}$ to show four distinct species on the surface: methanol physisorbed to the substrate, methanol chemisorbed to the substrate through a hydrogen bond to the oxygen ion in

* To whom correspondence should be addressed. Telephone: 1-617287-6096. FAX: 1-617-287-6066. E-mail: michelle.foster@umb.edu. the surface, and two reacted methoxy species. The first reacted species is bonded by a monodentate linkage through an oxygen atom to a magnesium ion, with an adjacent hydrogen atom forming a hydroxyl group with an exposed oxygen ion of the surface, resulting from the breaking of the $\mathrm{O}-\mathrm{H}$ bond in the adsorbed methanol. The second reacted species is bonded through a bidentate linkage via the methanol oxygen atom to two magnesium ions on the substrate. Recent theoretical investigations of methanol on $\mathrm{MgO}$ support these assignments. $7,16,17$

Under typical ultrahigh vacuum (UHV) conditions at both cryogenic temperatures ${ }^{7-12}$ and room temperature, ${ }^{11,12}$ methanol adsorption has also been studied on thin films of $\mathrm{MgO}(100)$ by exposing the substrate to a known pressure or coverage of methanol and then reestablishing UHV before examining the surface. For this reason, only the cryogenic studies showed any type of physisorption between the methanol and the $\mathrm{MgO}(100)$ surface with methanol wetting the surface and growth occurring in layers, that is, the methanol monolayer is saturated before multilayer formation begins. ${ }^{8,18}$ Any reaction occurring on the thin film $\mathrm{MgO}(100)$ substrate has been linked to defect sites such as terrace steps, edges, and vacancies on the surface..$^{7,11,12}$ When reactions were seen between methanol and the thin films of $\mathrm{MgO}(100)$, they occurred through cleavage of the $\mathrm{O}-\mathrm{H}$ bond in the methanol, forming a surface methoxide species similar to the monodentate linkage described above on $\mathrm{MgO}$ powders. ${ }^{7-12}$

Few studies have been performed to investigate methanol adsorption on single-crystal $\mathrm{MgO}(100)$ substrates, and they were always under UHV conditions. ${ }^{11,12}$ These investigations concur with those on the thin films of $\mathrm{MgO}(100)$ in that the atomically smooth terraces show no reaction with methanol. Only when the substrate has been intentionally damaged by sputtering is limited reactivity seen between methanol and the $\mathrm{MgO}(100)$ surface. It is important to reiterate that these studies were performed under UHV, nonequilibrium conditions and, until now, no studies have been performed under ambient conditions on the dynamic equilibrium occurring between methanol in the gas phase and the single-crystal $\mathrm{MgO}(100)$ surface. 
This paper describes a study that uses transmission Fourier transform infrared (FTIR) spectroscopy to study methanol on $\mathrm{MgO}(100)$ at room temperature and methanol pressures ranging from 0.1 Torr up to 105 Torr. In this manner, it is possible to investigate the surface interactions of adsorbed methanol in dynamic equilibrium with the gas phase on the $\mathrm{MgO}$ surface. Frequency shifts, bandwidths, and absorbance values of the $\mathrm{OH}$ stretching features are used to interpret the structure and phase of the interfacial region, giving both a qualitative and a quantitative understanding of methanol adlayers.

\section{Experimental Section}

For spectroscopic measurements, 16 crystals, cleaved along the (100) faces of $\mathrm{MgO}$ (MTI Corporation) under dry nitrogen purge, were placed in a cylindrical stainless steel sample cell that has been described elsewhere. ${ }^{19,20}$ Spacers made of $0.1 \mathrm{~mm}$ diameter Ta wire were sandwiched between the crystals so that the $\mathrm{MgO}$ faces did not touch. Calcium fluoride windows (Infrared Optical Products, Inc.) were attached to both ends of the cell and then sealed with Viton O-rings.

The cell was attached to a vacuum line and then pumped with a diffusion pump to a base pressure of $10^{-6}$ Torr. The cell and line were baked under vacuum at $110{ }^{\circ} \mathrm{C}$ for 2 days to remove contaminants. The methanol vapor was obtained from a liquid sample (Aldrich, HPLC grade) and further purified by a number of freeze-pump-thaw cycles. Methanol vapor was introduced at the desired pressure, and the dynamic equilibrium occurring between the methanol and the $\mathrm{MgO}(100)$ surfaces was monitored by transmission FTIR spectroscopy (Nicolet, Nexus 670) using $8 \mathrm{~cm}^{-1}$ resolution. An InSb detector was used during these studies that, coupled with the $\mathrm{CaF}_{2}$ windows, limits the frequency range of the FTIR spectra collected to 4500-1800 $\mathrm{cm}^{-1}$.

The $\mathrm{MgO}$ crystals were monitored, using transmission FTIR spectroscopy, for the presence of carbonaceous contamination in the form of a $\mathrm{C}-\mathrm{H}$ stretch at $\sim 3000 \mathrm{~cm}^{-1}$ (ref 21) and a $\mathrm{C}=\mathrm{O}$ stretch at $\sim 2200 \mathrm{~cm}^{-1}$ (ref 22). Concurrent studies using identically prepared crystals in a different cell and using $\mathrm{ZnSe}$ windows and an MCT detector allowed for the inspection of the crystals for the suspected reaction between $\mathrm{MgO}$ and $\mathrm{CO}_{2}$ to form $\mathrm{MgCO}_{3}$ by probing the spectra for the carbonate features, bands centered at 1660 and $1310 \mathrm{~cm}^{-1}$ (ref 23). At no time during this series of experiments were any carboncontaining features seen in any of the IR spectra. This is predictable in light of recent experiments on single-crystal $\mathrm{MgO}$, indicating that any reaction occurring between $\mathrm{MgO}(100)$ and $\mathrm{CO}_{2}$ is reversible. ${ }^{24}$ These results indicate that it is very unlikely that the surfaces of the crystals used in these experiments are contaminated with carbonaceous compounds.

For the methanol vapor spectroscopic investigations, a second sample cell was built using a quartz body ${ }^{25}$ press-fit against windows of $\mathrm{ZnSe}$ (Infrared Optical Products, Inc.), once again using Viton O-rings as the sealing medium. The combination of the MCT detector and the ZnSe windows allows the FTIR spectra to access a frequency range of $4000-800 \mathrm{~cm}^{-1}$. The methanol vapor was introduced into the cell by a method identical to the one described for the adsorption studies.

The ATR-FTIR spectra of liquid methanol (Aldrich, HPLC Grade) were taken by using a Nicolet Avatar spectrometer with a room-temperature DGTS detector and a germanium-window, single-bounce, attenuated total reflectance (ATR) accessory (Nicolet Omni Sampler) installed into the sample compartment.

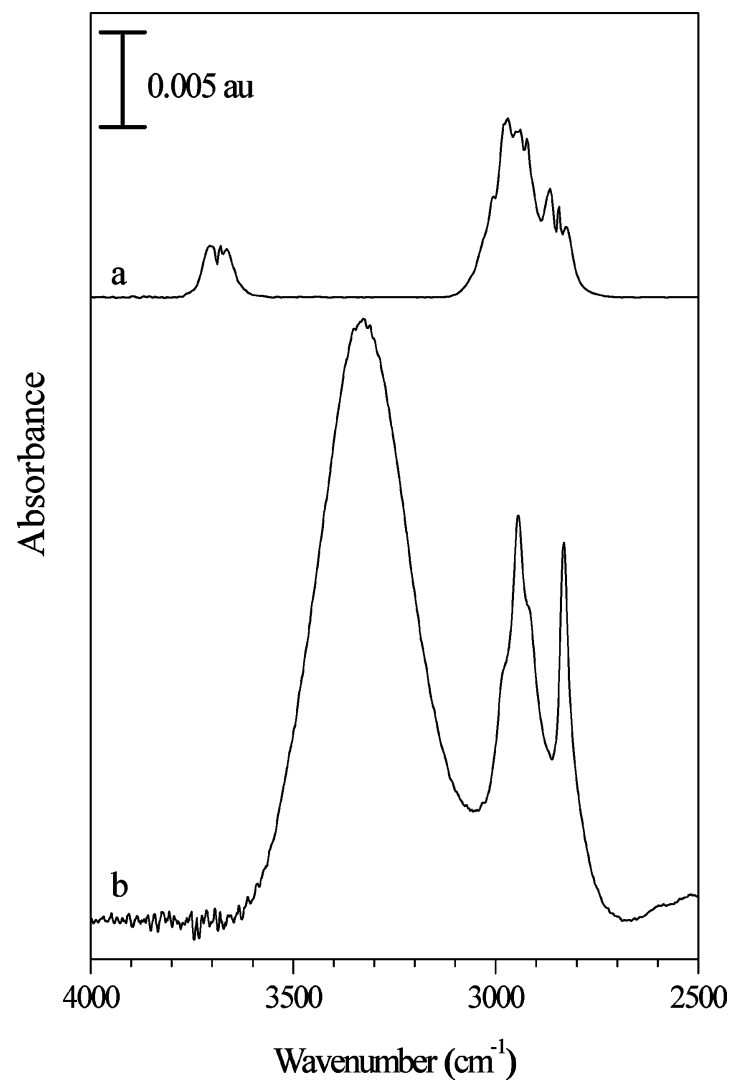

Figure 1. (a) FTIR spectra of 1.04-Torr methanol vapor and (b) ATRFTIR spectra of pure methanol liquid. Both spectra are sampling identical number densities of methanol, $1.7 \times 10^{17}$ molecules $/ \mathrm{cm}^{2}$.

\section{Results and Discussion}

1. Methanol Vapor. The $\mathrm{OH}$ stretch of methanol in an IR spectrum shifts according to the phase and hydrogen-bonding arrangement. Figure 1 shows the infrared absorption spectra of 1.04-Torr methanol vapor (top) and that of a drop of liquid methanol on the ATR crystal (bottom). The number of molecules examined for each of these spectra is nearly identical at $1.7 \times$ $10^{17}$ molecules $/ \mathrm{cm}^{2}$. The $\mathrm{OH}$ stretch appears at $3700 \mathrm{~cm}^{-1}$ for the vapor phase, while in the condensed phase the $\mathrm{OH}$ stretching feature is enhanced by a factor of 10 and red-shifted $350 \mathrm{~cm}^{-1}$ to approximately $3350 \mathrm{~cm}^{-1}$ as a function of the hydrogen bonding and condensation. The $\mathrm{CH}$ stretching features remain virtually unchanged at 2950 and $2830 \mathrm{~cm}^{-1}$, with a doubling of the intensity because of the condensation of methanol.

Methanol is very much like water in that its physical state, and thus the extent and the rigidity of its hydrogen bonding, can be investigated with FTIR spectroscopy. In previous studies, while investigating the interactions between water and $\mathrm{MgO}$ (100), ${ }^{19}$ the water vapor features in the FTIR spectra of water thin films on $\mathrm{MgO}(100)$ could be removed via subtraction using a reference water vapor spectrum. This procedure is possible because the $\mathrm{OH}$ stretching feature in the FTIR spectrum of water vapor does not vary as a function of pressure under ambient conditions (the vapor pressure of water is 17.6 Torr at $20^{\circ} \mathrm{C}$ ). This is not true of methanol vapor. The vapor pressure of methanol at ambient temperatures is an order of magnitude greater than that of water, 110 Torr at $22{ }^{\circ} \mathrm{C}$, and there are slight changes occurring in the spectra as a function of pressure. This can be seen in Figure 2a, which shows the $\mathrm{OH}$ stretching region for methanol vapor at pressures of 5, 25, 50, 75, and 100 Torr. This region of the spectrum is dominated by the monomer gas-phase features with its $\mathrm{OH}$ stretch appearing at 


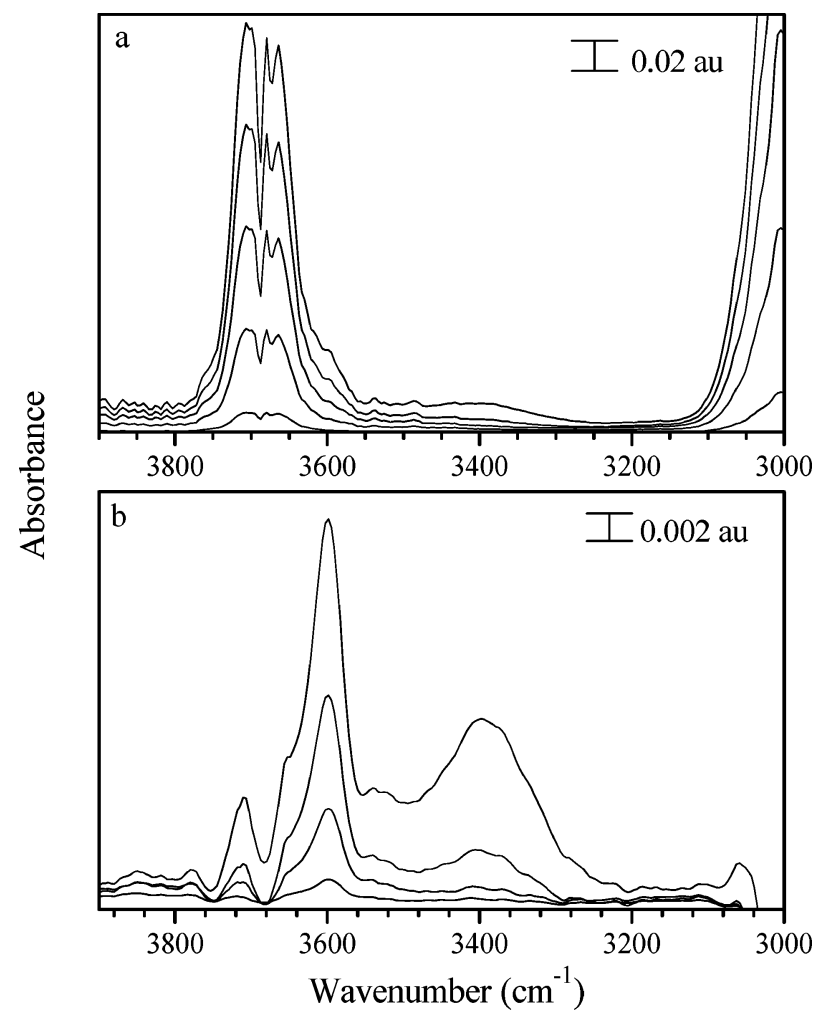

Figure 2. (a) FTIR spectra of methanol vapor at 5, 25, 50, 75, and 100 Torr (from bottom to top). (b) Gas-phase hydrogen bonding in methanol vapor, obtained by the subtraction of FTIR spectrum of 5-Torr methanol from spectra at 25, 50, 75, and 100 Torr (from bottom to top).

$\sim 3700 \mathrm{~cm}^{-1}$. However, there are also features appearing at lower wavelengths, a shoulder at $3600 \mathrm{~cm}^{-1}$ and a peak at 3400 $\mathrm{cm}^{-1}$, that cannot be explained by noninteracting gas-phase methanol molecules.

The spectra in Figure $2 \mathrm{~b}$ have been modified using a 5-Torr methanol vapor spectra to subtract out the monomer features, leaving only the peaks that may be attributed to hydrogenbonded methanol vapor. These features have been assigned in a recent investigation by Dixon et al. ${ }^{26}$ Using ab initio calculations and comparisons with dilute liquid solutions, the peak at $3600 \mathrm{~cm}^{-1}$ was assigned to an open methanol dimer, while the feature seen at $3400 \mathrm{~cm}^{-1}$ was due to higher-order methanol polymers, probably trimers and/or tetramers in a cyclic configuration. No attempt was made to quantify the hydrogenbonded methanol.

The quartz cell used for the methanol vapor studies has a known path length $(5.00 \pm 0.05 \mathrm{~cm})$, and using this value in a Beer's Law calculation, along with the integrated areas of both the non-hydrogen-bonding $\mathrm{OH}$ peak at $\sim 3700 \mathrm{~cm}^{-1}$ and the $\mathrm{CH}$ feature centered at $2900 \mathrm{~cm}^{-1}$ for a number of methanol vapor FTIR spectra at pressures lower than 5 Torr, values were calculated for the molecular cross sections for methanol using these two features. For the $\mathrm{CH}$ stretching region (integrated from 2725 to $3140 \mathrm{~cm}^{-1}$ ) the molecular cross section, $\bar{\sigma}_{g, \mathrm{CH}}$, was calculated to be $9.7 \times 10^{-18} \mathrm{~cm} /$ molecule. For the monomeric $\mathrm{OH}$ stretching region (assuming there is minimal hydrogen bonding occurring in the gas phase at these low pressures) integrated from 3560 to $3780 \mathrm{~cm}^{-1}$, the molecular cross section, $\bar{\sigma}_{g, \mathrm{OH}}$, was calculated to be $1.6 \times 10^{-18} \mathrm{~cm} / \mathrm{molecule}$. These values were then used to calculate the concentration of the methanol vapor present at pressures up to 105 Torr. The difference between the methanol concentrations calculated from the $\mathrm{CH}$ stretch (the actual methanol concentration) and the $\mathrm{OH}$

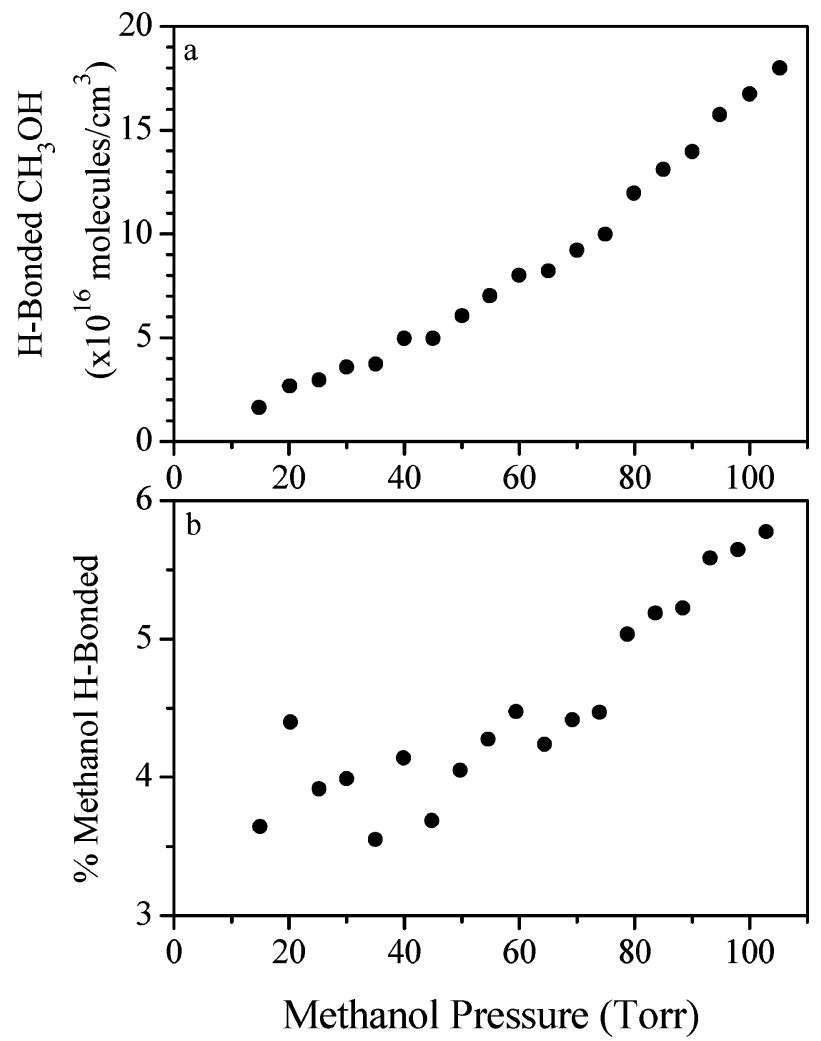

Figure 3. (a) Concentration of methanol molecules hydrogen bonding in the gas phase as a function of methanol pressure, calculated as described in the text. (b) The number of methanol molecules undergoing hydrogen bonding presented as a percentage of the total number of methanol molecules present.

monomeric stretch (the concentration of methanol monomers) gives a rough estimate of the concentration of methanol molecules undergoing hydrogen bonding.

This concentration of hydrogen-bonded methanol molecules as a function of total methanol pressure is presented in Figure $3 \mathrm{a}$. If the hydrogen-bonding arrangement in the gas phase is the same as that calculated by Dixon et al. ${ }^{26}$ namely an open dimer and cyclic tetramer, then the concentration of methanol monomers calculated from the integrated area of the feature at $3700 \mathrm{~cm}^{-1}$ includes both true monomers and one-half of every dimer pair, and thus is a high estimate. This means that the calculated value of the concentration of molecules hydrogen bonding in the gas phase is a low estimate. Attempts to use these data to calculate the concentration of methanol dimers versus the molecules hydrogen bonding in larger clusters have been unsuccessful, since the two configurations of hydrogenbonded clusters have led to drastically different molecular cross sections for the $\mathrm{OH}$ stretch.

Figure $3 \mathrm{~b}$ presents this same calculation in terms of the percentage of molecules participating in hydrogen bonding, the ratio of the difference between methanol concentrations calculated using the $\mathrm{CH}$ stretch peak, and the $\mathrm{OH}$ stretch feature with respect to the total concentration of methanol present, multiplied by 100 . It is interesting to note that at pressures below 55 Torr, the percent of hydrogen-bonded methanol molecules is fairly constant at about $4 \%$ with a slight increase with increasing pressure; yet, at methanol pressures of 60 Torr up to 105 Torr, there is a notable increase in the percentage of hydrogen-bonded methanol.

This fact is very important in the current study, which investigates the interactions occurring between the $\mathrm{MgO}(100)$ surface and many different pressures of methanol vapor during 


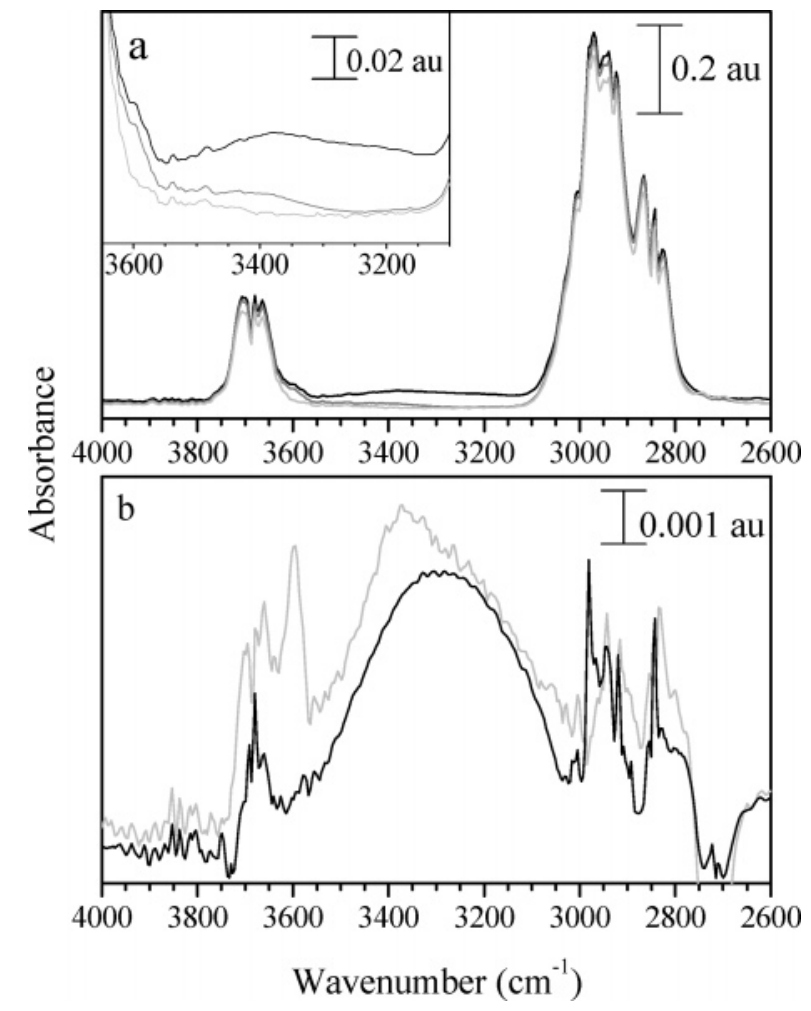

Figure 4. (a) FTIR spectra of 5-Torr methanol vapor (light-gray spectrum on bottom, $\times 17$ ), 90-Torr methanol vapor (dark-gray spectrum in middle), and 90-Torr methanol in equilibrium with $16 \mathrm{MgO}(100)$ crystals (black spectrum on top, $\times 5$ ). (Inset) Enlarged view of the hydrogen-bonded $\mathrm{OH}$ stretching region of the same three spectra. (b) Subtraction result of 90-Torr methanol in association with $\mathrm{MgO}(100)$, $n=32$, using both the 90-Torr methanol vapor spectrum (black) and the 5-Torr methanol spectrum (light gray) as a reference spectra for removal of the vapor features.

dynamic equilibrium. The presence of hydrogen bonding in the FTIR spectrum is not enough to distinguish between methanol in the vapor phase and methanol condensed on our substrate. When investigating the interaction between a model surface and any molecule at elevated pressures, extensive knowledge of how that molecule behaves when the substrate is absent is essential.

2. Methanol and Magnesium Oxide. The experimental apparatus used for this study has been designed to minimize the number of molecules in the gas phase probed during our adsorption studies. However, even with these extreme measures, the signal from the gas-phase methanol drowns out the signal from adsorbed methanol, as can be clearly seen in Figure 4a; the black spectrum is that of 90 Torr of methanol in the presence of $16 \mathrm{MgO}(100)$ crystals. The adsorbed methanol is represented by the slight mound appearing in this spectrum at approximately $3300 \mathrm{~cm}^{-1}$. However, because of the residual gas-phase methanol in the sample cell, it is difficult to quantify this adsorbed feature. The residual gas-phase features can be subtracted out of this spectrum, leaving only those features representative of the adsorbed methanol adlayer. Typically in this type of investigation, ${ }^{19,27-31}$ a low-pressure, vapor-phase reference spectrum is chosen and used to subtract the vapor features from all the resulting sample spectra. For instance, the 5-Torr spectrum, shown in light gray in Figure $4 \mathrm{a}$, could be used to remove the vapor features from the 90-Torr $\mathrm{MeOH} /$ $\mathrm{MgO}(100)$ sample spectrum, resulting in the light-gray adsorption spectrum shown in Figure 4b. However, because of the hydrogen bonding of methanol in the vapor phase, the resulting subtraction spectrum shows not only the features associated with methanol adsorbed to $\mathrm{MgO}(100)$ when at equilibrium with the vapor but also the hydrogen bonding occurring in the gas phase, as indicated by the sharp feature at $3600 \mathrm{~cm}^{-1}$ and the asymmetry of the adsorbed feature culminating in a peak at 3400 $\mathrm{cm}^{-1}$. By comparing this subtraction result with the spectra in Figure $2 b$, it is clear that these two features are due to methanol vapor.

Figure 4a also shows the gas-phase FTIR spectrum of 90 Torr of methanol, indicated in dark gray. In the full-scale versions of all three spectra, it is difficult to see any differences, but the inset shows the hydrogen-bonding region for all three spectra, 5-Torr methanol vapor, 90-Torr methanol vapor, and 90-Torr methanol vapor in the presence of $16 \mathrm{MgO}$ crystals. In this expanded graph, it is easy to see the differences and similarities of the three spectra, most notably a shoulder at $3600 \mathrm{~cm}^{-1}$ and the feature at $3400 \mathrm{~cm}^{-1}$, which are absent in the 5-Torr methanol vapor spectrum. When the 90 -Torr methanol vapor spectrum is used as a reference for the subtraction of the vapor features from the spectrum of 90-Torr methanol in equilibrium with the $\mathrm{MgO}(100)$ surfaces, the black spectrum in Figure $4 \mathrm{~b}$ is the result. The peak associated with the $\mathrm{CH}$ stretches can be seen between 2750 and $3050 \mathrm{~cm}^{-1}$; these features are due to both adsorbed methyl groups on the surface and subtraction artifacts. Likewise, the feature centered at $3690 \mathrm{~cm}^{-1}$ is most likely due to gas-phase methanol and a result of poor subtraction; however, it could also be due to either non-hydrogen-bonded adsorbed methanol or the reversible dissociation of methanol on the surface. The peak of interest is the very broad feature, with a full width at half-maximum (fwhm) of $400 \mathrm{~cm}^{-1}$, centered at $3300 \mathrm{~cm}^{-1}$. This feature, which strongly resembles that of liquid methanol, is the $\mathrm{OH}$ stretching vibration of methanol adsorbed on $\mathrm{MgO}(100)$.

Absorption spectra of methanol adsorbed to $\mathrm{MgO}(100)$ at room temperature for a variety of pressures are shown in Figure 5. The bands are all broad, and the fwhm slightly decreases with increasing pressure, from a value of $460 \mathrm{~cm}^{-1}$ at 5 Torr to $390 \mathrm{~cm}^{-1}$ at 105 Torr. The peak center of the $\mathrm{OH}$ stretching feature of the adsorbed methanol also shifts as a function of coverage. At the lower pressures, the peak center is relatively constant at $3260 \pm 10 \mathrm{~cm}^{-1}$; but beginning at approximately 70 Torr, the peak center becomes increasingly blue-shifted until, at 105 Torr, the highest pressure investigated in this study, the peak center occurs at $3330 \mathrm{~cm}^{-1}$. Figure 6 shows this peak shift trend as a function of methanol vapor pressure. The low-pressure methanol peak centers are close to the peak center seen for vitreous solid methanol cooled to $-180^{\circ} \mathrm{C}$; this feature has been observed at $3235 \mathrm{~cm}^{-1}$ (ref 32). At approximately 70 Torr, the peak center begins to shift toward the $3337 \mathrm{~cm}^{-1}$ observed for bulk liquid methanol. ${ }^{32}$ This seems to indicate that, at the lower pressures, the molecules are oriented in such a way that their hydrogen-bonding network is more solidlike; whereas as the pressure increases, the intermolecular interactions become decidedly more liquidlike.

Since the high-pressure absorption resembles that of bulk liquid methanol, its optical properties were used to estimate adlayer coverage. By making use of a modified Beer-Lambert relationship, ${ }^{33,34}$ the integrated absorbance of a surface species is described by the following equation:

$$
\bar{A}=\frac{n \bar{\sigma} S_{\mathrm{CH}_{3} \mathrm{OH}}}{2.303}
$$

The integrated absorbance, $\bar{A}\left(\mathrm{~cm}^{-1}\right)$, of the adsorbed methanol was determined by numerical integration over the frequency range $3630-3030 \mathrm{~cm}^{-1}, S_{\mathrm{CH}_{3} \mathrm{OH}}$ is the surface density of 


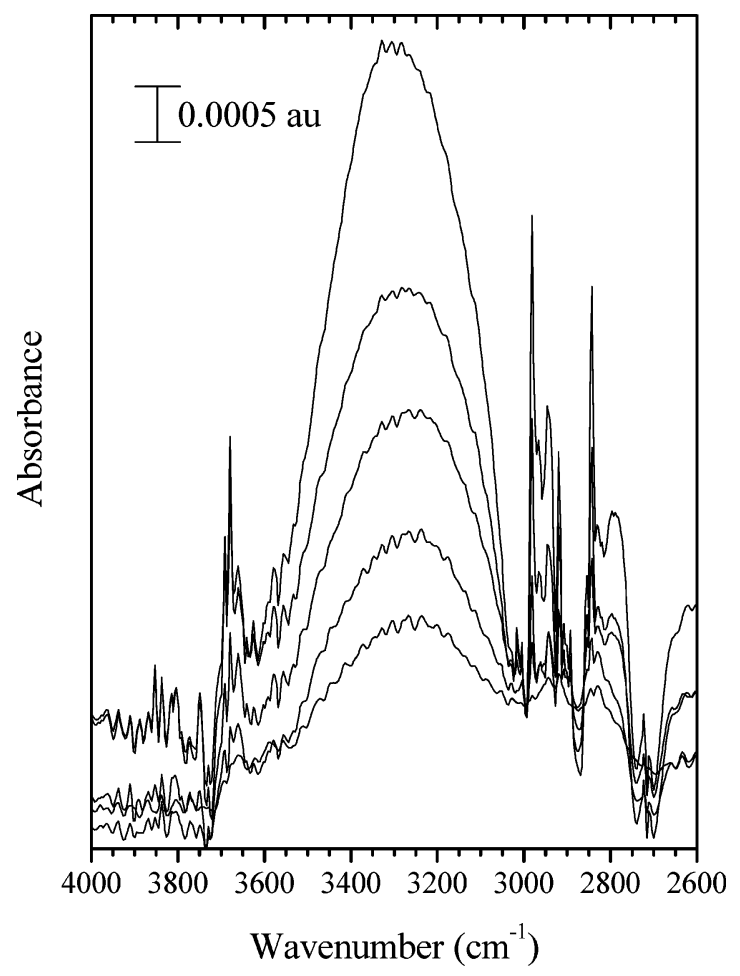

Figure 5. Absorption spectra of 5, 25, 50, 75, and 100 Torr (from bottom to top) of methanol adsorbed on $\mathrm{MgO}(100)$ at room temperature. These spectra correspond, respectively, to $0.45,0.70,0.90,1.05$, and 1.50 monolayers of methanol adsorbed to the $\mathrm{MgO}(100)$ surface. The number of $\mathrm{MgO}(100)$ crystal faces for these experiments is $n=32$. Gas-phase features have been removed from these spectra by using the corresponding pressure of methanol vapor spectrum.

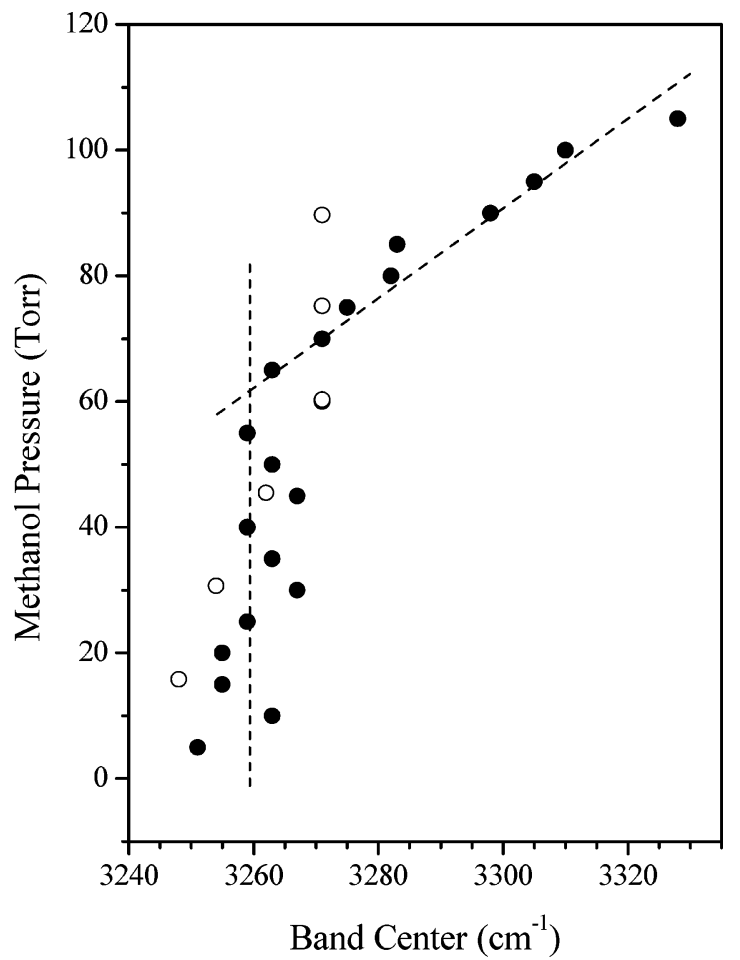

Figure 6. Band center change as a function of methanol pressure in equilibrium with $\mathrm{MgO}(100)$ at room temperature. Data taken on ascending and descending pressures are given by the closed and open circles, respectively. The lines are merely a guide for the eye.

adsorbed methanol, and $n$ is the number of exposed $\mathrm{MgO}(100)$ faces, 32 for this series of experiments. The integrated cross section, $\bar{\sigma}(\mathrm{cm} / \mathrm{molecule})$, was determined from the measured, frequency-dependent, imaginary component, $\kappa$, of the index of refraction of bulk methanol ${ }^{35}$ using: ${ }^{36}$

$$
\bar{\sigma}=\left(\frac{4 \pi}{\rho}\right) \int_{\text {band }} \kappa \tilde{v} \mathrm{~d} \tilde{v}
$$

where $\rho$ is the molecular density of methanol at the given temperature. For methanol at room temperature, $\bar{\sigma}$ was found to be $1.02 \times 10^{-16} \mathrm{~cm} / \mathrm{molecule}$. Once $S_{\mathrm{CH}_{3} \mathrm{OH}}$ is calculated, the coverage, $\Theta=S_{\mathrm{CH}_{3} \mathrm{OH}} / S_{\mathrm{MgO}}$, is easily determined since $S_{\mathrm{MgO}}$ for the $\mathrm{MgO}(100)$ face is $1.13 \times 10^{15}$ surface ions $/ \mathrm{cm}^{2}$ (ref 37). Using this procedure, all integrated absorbances are converted to coverages, $\Theta$, in terms of methanol molecules per surface ion. Using this method of analysis, the methanol adlayer shown in Figure 4b corresponds to a coverage of 1.3 monolayers.

While we can justify the use of liquid methanol optical constants to calculate coverages when the band profiles of the bulk liquid and adlayers are similar, there is a systematic error introduced when the profiles differ as they do in the lower coverage features, which we shall associate with the submonolayer adlayer. It would be useful to make use of the optical properties of solid methanol for the low-pressure regime; however, those proved difficult to find in the literature, and thus, all calculations were made using the optical constants of liquid methanol. By comparing the properties of water, whose optical constants are very well represented in the literature, ${ }^{38-40}$ with those of methanol, an estimation of the error associated with our calculations might be possible. The optical qualities of methanol and water are similar upon transition from the vapor to the liquid phase, namely, the myriad rotational-vibrational features of the gas transform into a single diffuse absorption band with a corresponding increase by a factor of 10 in the oscillator strength of the $\mathrm{OH}$ bond because of the hydrogen bonding within the system. ${ }^{35,41}$ Therefore, perhaps the optical qualities of water during the liquid-to-solid transition may be used as a guide; liquid water has a peak center at $3400 \mathrm{~cm}^{-1}$ (ref 39), which shifts to $3250 \mathrm{~cm}^{-1}$ (ref 40) upon condensation to ice, while the oscillator strength of the $\mathrm{OH}$ stretch doubles. In correlations between the integrated cross section and band center for hydrogen-bonding systems just reviewed, we anticipate higher values of $\bar{\sigma}$ for the lower vibrational frequency of the low-coverage region, and thus, calculated coverage values are most likely underestimated by as much as $50 \%$.

Isotherms may be determined from the pressure-dependent adlayer spectra through the photometry analysis just described. By using the spectra shown in Figure 5 and others as well, the room temperature adsorption isotherm for methanol on $\mathrm{MgO}(100)$ was calculated and is shown in Figure 7. The methanol coverage is plotted as a function of the methanol pressure at equilibrium within the cell. In this figure, the closed circles represent adsorption measurements, and the open circles are the desorption measurements. The highest pressure used in this series of experiments was 105 Torr. There is significant hysteresis shown in the isotherm. This hysteresis is not permanent, and the substrate is returned to virtually its initial state after being evacuated overnight. The reactivity between methanol and $\mathrm{MgO}(100)$ at high pressures and room temperature was recently investigated by this researcher using atomic force microscopy, and there were no topographical changes recorded when $\mathrm{MgO}(100)$ was allowed to sit in an atmosphere of pure methanol vapor for $100 \mathrm{~h} .{ }^{20}$

According to the adsorption isotherm presented in Figure 7, a monolayer of methanol on $\mathrm{MgO}(100)$ occurs at a pressure of 70 Torr. We have defined one monolayer as a single methanol molecule adsorbed onto every available surface ion, both $\mathrm{Mg}^{2+}$ 


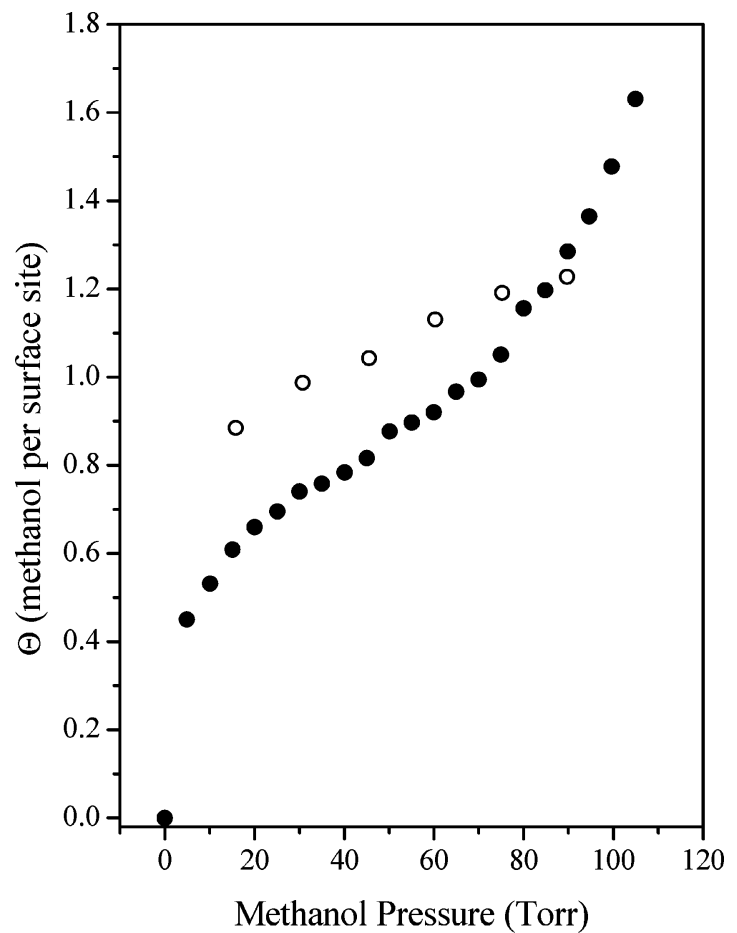

Figure 7. Adsorption isotherm of methanol on $\mathrm{MgO}(100)$ at room temperature. Data taken on ascending and descending pressures are given by closed and open circles, respectively.

and $\mathrm{O}^{2-}$. At pressures above 70 Torr, multilayers of methanol begin to form on the substrate. The crystal structure of $\mathrm{MgO}(100)$ has a cation interatomic distance of $2.8 \AA, 42$ which is a close match to the hydrogen-bonding oxygen interatomic distance of $3.0 \AA^{43}$ seen in hexagonal ice, and is most likely a distance that will allow the methanol molecule to interact with a surface ion and have the added stability of hydrogen bonding with a neighboring methanol molecule. Once all the surface sites have been filled, the impinging methanol molecule adsorbs onto this methanol monolayer, creating a second layer. Even at methanol pressures as high as 105 Torr, the methanol adlayer is minimal, with 1.63 layers being the maximum film thickness observed in this investigation. Once the methanol molecules begin three-dimensional growth on the surface, the hydrogenbonding network in the adlayer loosens up and becomes more liquidlike.

This supposition most likely explains the shift in band center that begins at a pressure of 70 Torr, as seen in Figure 6. The correlation between coverage and band center is better revealed by Figure 8, which plots the band center of the adsorbed methanol hydroxyl stretch as a function of coverage. At coverages below one monolayer, the band is centered at 3260 $\mathrm{cm}^{-1}$, but once the monolayer is complete and multilayer formation begins, the peak center begins to shift to the lower wavenumbers. As discussed earlier, the peak center seen for coverages of one monolayer and below closely resembles that of solid methanol. This tells us that, at coverages of one monolayer of methanol and below, the molecules are linked by a very rigid hydrogen-bonding network to the substrate. This monolayer seems to be quite stable as demonstrated by the coverage and peak center of a desorption series, represented by the open circles in Figures 6-8. At pressures as high as 90 Torr and as low as 15 Torr during a desorption series, the coverage remains fairly constant at one monolayer, and the band center falls within the submonolayer regime. Once multilayer growth begins, the hydrogen-bonding network becomes looser and more liquidlike as the adlayer grows in three dimensions.

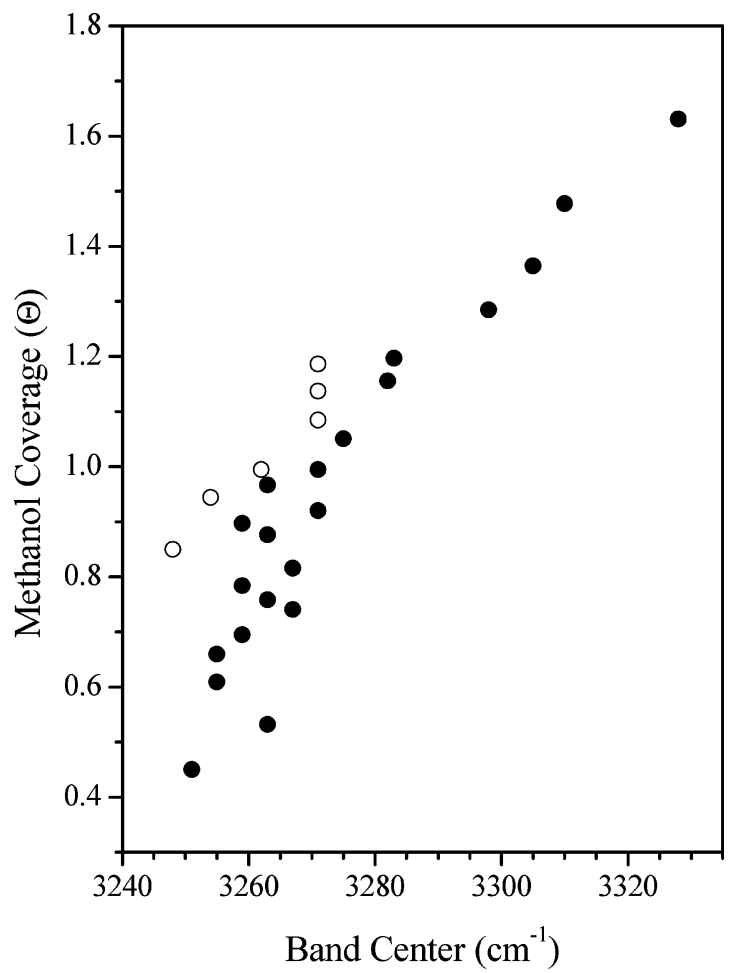

Figure 8. Band center change with methanol coverages on $\mathrm{MgO}(100)$ at room temperature. Data taken on ascending and descending pressures are given by closed and open circles, respectively.

\section{Conclusions}

Methanol molecularly adsorbs to the magnesium oxide surface, forming reversible thin films at room temperature and under moderate-to-high methanol pressures. Film growth occurs by layers, with the completion of the monolayer before multilayer growth begins. At submonolayer coverages, the methanol forms a rigid, solidlike hydrogen-bonding network, while at multilayer coverages, the methanol adlayer has a liquidlike hydrogen-bonding network. The monolayer of methanol on $\mathrm{MgO}(100)$ is a fairly stable structure, as shown by the hysteresis seen during a full adsorption/desorption series. The maximum coverage obtained was less than two layers at a methanol vapor pressure of 105 Torr. All of the thin films discussed in this investigation are the result of the dynamic equilibrium occurring between methanol in the gas phase and a series of $\mathrm{MgO}(100)$ crystals.

Acknowledgment is made to the donors of the Petroleum Research Fund, administered by the American Chemical Society, for partial support of this research. This research was also supported by an award from Research Corporation.

\section{References and Notes}

(1) Onishi, H.; Egawa, C.; Aruga, T.; Iwasawa, Y. Surf. Sci. 1987, 191,479

(2) Adsorption on Ordered Surfaces of Ionic Solids and Thin Films; Freund, H.-J.; Umbach, E., Eds.; Springer-Verlag: Berlin, 1993.

(3) Noguera, C. Physics and Chemistry at Oxide Surfaces; Cambridge: Cambridge, 1996.

(4) Tench, A. J.; Giles, D.; Kibblewhite, J. F. J. Trans. Faraday Soc. 1971, 67, 854.

(5) Foyt, D. C.; White, J. M. J. Catal. 1977, 47, 260.

(6) Bensitel, M.; Saur, O.; Lavalley, J. C. Mater. Chem. Phys. 1991, 28,309

(7) Di Valentin, C.; Del Vitto, A.; Pacchioni, G.; Abbet, S.; Worz, A. S.; Judai, K.; Heiz, U. J. Phys. Chem. B 2002, 106, 11961.

(8) Gunster, J.; Liu, G.; Stultz, J.; Krischok, S.; Goodman, D. W. J. Phys. Chem. B 2000, 104, 5738 . 
(9) Goodman, D. W. J. Vac. Sci. Technol., A 1996, 14, 1526.

(10) Wu, M.-C.; Estrada, C. A.; Corneille, J. S.; Goodman, D. W. J. Chem. Phys. 1992, 96, 3892.

(11) Peng, X. D.; Barteau, M. A. Langmuir 1991, 7, 1426.

(12) Peng, X. D.; Barteau, M. A. Catal. Lett. 1992, 12, 245.

(13) Li, C.; Li, G.; Xin, Q. J. Phys. Chem. 1994, 98, 1933.

(14) Heidberg, J.; Redlich, B.; Wetter, D. Ber. Bunsen-Ges. Phys. Chem. 1995, 99, 1333 .

(15) Liang, S. H. C.; Gay, I. D. Langmuir 1985, 1, 593.

(16) Branda, M. M.; Peralta, J. E.; Castellani, N. J.; Contreras, R. H. Surf. Sci. 2002, 504, 235.

(17) Branda, M. M.; Ferullo, R. M.; Belelli, N. J.; Castellani, N. J. Surf. Sci. 2003, 527, 89.

(18) Gunster, J.; Liu, J.; Stultz, J.; Goodman, D. W. J. Chem. Phys. 1999, $110,2558$.

(19) Foster, M.; Furse, M.; Passno, D. Surf. Sci. 2002, 502-503, 102.

(20) Foster, M.; Passno, D.; Rudberg, J. J. Vac. Sci. Technol., A 2004, 22,1640 .

(21) Simanouchi, T. Tables of Molecular Vibrational Frequencies; American Chemical Society: New York, 1972.

(22) Heidberg, J.; Redlich, B. J. Electron Spectrosc. Relat. Phenom. 1993, 64/65, 599

(23) Kohno, Y.; Ishikawa, H.; Tanaka, T.; Funabiki, T.; Yoshida, S. Phys. Chem. Chem. Phys. 2001, 3, 1108.

(24) Carrier, X.; Doyle, C. S.; Kendelewicz, T.; Brown, G. E., Jr. Surf. Rev. Lett. 1999, 6, 1237.

(25) Peters, S. J.; Ewing, G. E. J. Phys. Chem. B 1997, 101, 10880.

(26) Dixon, J. R.; George, W. O.; Hossain, M. F.; Lewis, R.; Price, J. M. J. Chem. Soc., Faraday Trans. 1997, 93, 3611.
(27) Foster, M.; Ewing, G. E. J. Chem. Phys. 2000, 112, 6817.

(28) Al-Abadleh, H.; Grassian, V. H. Langmuir 2003, 19, 341.

(29) Al-Abadleh, H.; Grassian, V. H. J. Phys. Chem. B 2003, 107, 10829.

(30) Underwood, G. M.; Miller, T. M.; Grassian, V. H. J. Phys. Chem. A 1999, 103, 6184.

(31) Goodman, A. L.; Bernard, E. T.; Grassian, V. H. J. Phys. Chem. A 2001, 105, 6443 .

(32) Falk, M.; Whalley, E. J. Chem. Phys. 1961, 34, 1554.

(33) Richardson, H. H.; Chang, H.-C.; Noda, C.; Ewing, G. E. Surf. Sci. 1989, 216, 93.

(34) Berg, O.; Ewing, G. E. Surf. Sci. 1989, 220, 207.

(35) Bertie, J. E.; Zhang, S. L.; Eysel, H. H.; Baluja, S.; Ahmed, M. K. Appl. Spectrosc. 1993, 47, 1100.

(36) Weis, D. D.; Ewing, G. E. J. Geophys. Res. [Atmos.] 1996, 101, 18709

(37) Henrich, V. E.; Cox, P. A. The Surface Science of Metal Oxides; Cambridge University Press: Cambridge, 1996.

(38) Bertie, J. E.; Ahmed, M. K.; Eysel, H. H. J. Phys. Chem. 1989, 93, 2210 .

(39) Downing, H. D.; Williams, D. J. Geophys. Res. 1975, 80, 1656.

(40) Warren, S. G. Appl. Opt. 1984, 23, 1206.

(41) Fujita, Y.; Ikawa, S.-I. Chem. Phys. Lett. 1989, 159, 184

(42) CRC Handbook of Chemistry and Physics, 82nd ed.; Linde, D. R. Ed.; CRC Press: Boca Raton, FL, 2002; pp F167 and F169.

(43) Pimentel, G. C.; McClelland, A. L. The Hydrogen Bond; Reinhold: New York, 1960 